\title{
CORRECTION
}

\section{Correction to: Intracerebral Hemorrhage in COVID-19 Patients with Pulmonary Failure: A Propensity Score-Matched Registry Study}

Corinna N. Lang ${ }^{1,2^{*}}$, Johanna S. Dettinger ${ }^{1,2}$, Michael Berchtold-Herz ${ }^{3}$, Stefan Utzolino ${ }^{4}$, Xavier Bemtgen ${ }^{1,2}$, Viviane Zotzmann ${ }^{1,2}$, Bonaventura Schmid ${ }^{5}$, Paul M. Biever ${ }^{1,2}$, Christoph Bode ${ }^{1,2}$, Katharina Müller-Peltzer ${ }^{6}$, Daniel Duerschmied ${ }^{1,2}$, Tobias Wengenmayer ${ }^{1,2}$, Wolf-Dirk Niesen ${ }^{7 \dagger}$ and Dawid L. Staudacher ${ }^{1,2 \dagger}$

๑ 2021 Springer Science+Business Media, LLC, part of Springer Nature and Neurocritical Care Society

\section{Correction to: Neurocrit Care https://doi.org/10. 1007/s12028-021-01202-7}

This abstract of this article was updated to correct the following sentence to read: $\mathrm{ICH}$ was detected in 22 patients (13.5\%) with no statistical difference between the groups (11.2 vs. $19.1 \%$ without and with SARS-CoV-2, respectively, $\mathrm{p}=0.21$ ). Propensity score matching confirmed similar rates of ICH in both groups (12.8 vs. $19.1 \%$ without and with SARS-CoV-2, respectively, $\mathrm{p}=0.57$ ), thus leveling out possible confounders.
Author details

${ }^{1}$ Department of Cardiology and Angiology I, Faculty of Medicine, Heart Center Freiburg University, University of Freiburg, Hugstetter Str. 55, 79106 Freiburg, Germany. ${ }^{2}$ Department of Medicine III (Interdisciplinary Medical Intensive Care), Medical Center, Faculty of Medicine, University of Freiburg, Freiburg, Germany. ${ }^{3}$ Department of Cardiovascular Surgery, Faculty of Medicine, Heart Center Freiburg University, University of Freiburg, Freiburg, Germany.

${ }^{4}$ Department of General and Visceral Surgery, Medical Center, University of Freiburg, Freiburg, Germany. ${ }^{5}$ Department of Emergency Medicine, Faculty of Medicine, University of Freiburg, Freiburg, Germany. ${ }^{6}$ Department of Radiology, Faculty of Medicine, University of Freiburg, Freiburg, Germany. ${ }^{7}$ Department of Neurology, Faculty of Medicine, University of Freiburg, Freiburg, Germany. Publisher's Note

Springer Nature remains neutral with regard to jurisdictional claims in published maps and institutional affiliations.

Published online: 12 April 2021

*Correspondence: corinna.nadine.lang@uniklinik-freiburg.de

${ }^{\dagger}$ Wolf-Dirk Niesen and Dawid L. Staudacher have contributed equally.

${ }^{1}$ Department of Cardiology and Angiology I, Faculty of Medicine, Heart

Center Freiburg University, University of Freiburg, Hugstetter Str. 55,

79106 Freiburg, Germany

Full list of author information is available at the end of the article

The original article can be found online at https://doi.org/10.1007/ s12028-021-01202-7.

\section{实

